\title{
Back injuries to fast bowlers in cricket: a prospective study
}

\author{
D. Foster, Med, D. John, BPE(Hons), B. Elliott, PhD, T. Ackland, PhD and K. Fitch, MD
}

Department of Human Movement and Recreational Studies, The University of Western Australia

Eighty-two high performance young male fast bowlers (mean age $\mathbf{1 6 . 8}$ years) were tested immediately prior to the season for selected kinanthropometric and physiological data. Subjects were also filmed both laterally $(200 \mathrm{~Hz})$ and from above $(100 \mathrm{~Hz})$ while bowling so that their front foot impacted a force platform during the delivery stride. The players then completed a log book over the ensuing season that detailed their training and playing programmes. All cricket related injuries over this season were assessed by a sports physician who used computerized tomography to assist in the diagnosis of spinal injuries.

At the completion of this season the players were grouped according to their injury status (Group 1-bony injury to a vertebra; Group 2 - soft tissue injury to the back that caused the player to miss at least one game, and Group 3 - no injuries). A one-way analysis of variance was used to identify if any variables were significantly $(P<0.05)$ different between the three groups, and a Scheffe post hoc comparison was used to determine which groups were significantly different.

Eleven per cent of the players sustained a stress fracture to a vertebra(e) (L4 to S1), while 27 per cent sustained a soft tissue injury to the back. Bowlers with a low longitudinal foot arch were more likely to develop a stress fracture than those with a high arch. Shoulder depression and horizontal flexion strength for the preferred limb and quadriceps power in the non-preferred limb were also significantly related to back injuries. Bowlers who rotated the trunk to re-align the shoulders by more than $40^{\circ}$ to a more side-on position between back foot impact and front foot impact in the delivery stride were more likely to sustain back injuries. No significant relationship was determined between peak vertical (5.4 BW) or horizontal ( $-2.5 \mathrm{BW})$ ground reaction forces at front foot impact. However, these forces may predispose a bowler to back injuries if he bowls for long periods. Nineteen of 32 players (59 per cent) who bowled in excess of the mean number of matches for the group, suffered a stress fracture or soft tissue injury to the back compared to the 38 per cent injury frequency for the total group. A greater release height when expressed as a percentage of standing height was also significantly related to back injuries. Results suggest that bowlers with the above physical characteristics, who bowl with these biomechanical techniques for extended periods, are predisposed to back injuries.

Keywords: Cricket, fast bowling, sports injury, biomechanics, kinanthropometry

Address for correspondence: Dr B. Elliott, Department of Human Movement and Recreational Studies, The University of Western Australia, Nedlands, 6009 Western Australia

(C) 1989 Butterworth \& Co (Publishers) Ltd 0306-3674/89/030150-05 $\$ 03.00$
Increasing participation of children and adolescents in organized sport and fitness activities has resulted in an increased number of reported injuries ${ }^{1}$. Furthermore, new injuries, specific to certain sports, have been reported in these young athletes. Fast bowling, an integral part of the game of cricket, is an activity that in Australia has produced injuries to young fast bowlers in almost epidemic proportions. The young fast bowler has been described as the cricketer most susceptible to either traumatic or over-use injuries ${ }^{2}$. We have reported that 50 per cent of a sample of fast bowlers from one A grade club experienced a stress fracture over a five year period. Back injuries are commonest in activities requiring repetitive flexion, extension and/or rotation of the spine, e.g. gymnastics, high jumping, tennis serving and fast bowling ${ }^{1,3,4}$. This prospective study investigated the relationship between back injuries in cricket with the biomechanical techniques used in the bowling action and the physiological and kinanthropometric characteristics of young fast bowlers.

\section{Methods and procedures}

Eighty-two potentially high performance injury-free male fast bowlers, with a mean age of 16.8 years (range 15 to 22 years) were the subjects of this study. They were nominated by coaches as being the best young bowlers from club and/or school teams.

\section{Methods}

Subjects were tested two weeks prior to the 1986-1987 cricket season. Body mass was recorded on an Avery beam balance scale, and skinfold levels from the preferred side were measured at triceps, subscapular, supra-iliac, abdominal and calf sites using skinfold calipers ${ }^{5}$. Proportionality and body bulk were assessed for each subject by recording upper limb length, arm and forearm lengths, lower limb length, leg length, humerus width, femur width, biceps muscle girth and calf girth using techniques reported by Behnke and Wilmore ${ }^{6}$.

Hamstring and quadricep muscle group torque were measured using a Cybex II Isokinetic Dynamometer at a speed setting of 60 degrees per second. The highest score from three trials was used, firstly from the preferred and then the non-preferred 
limbs. The best score from three trials for shoulder depression, shoulder horizontal flexion, trunk flexion and trunk extension strengths was recorded from the preferred and non-preferred sides of the body using procedures described by Clarke and Clarke ${ }^{7}$. Shoulder and hamstring muscle group flexibility were measured on the preferred and non-preferred limbs using procedures outlined by Leighton ${ }^{8}$. Abdominal muscular endurance was assessed by a 60 second sit up test (AAHPER Youth Fitness Test) ${ }^{9}$. A photograph with a lateral and frontal view was then taken of each subject so that a posture assessment could be derived by an experienced tester based on the New York State Physical Fitness Test ${ }^{10}$. Each player's aerobic capacity was estimated by a 15 minute run ${ }^{11}$ at a separate testing session.

Each subject was appropriately marked for digitizing, warmed up thoroughly and then bowled three maximum velocity trials at a wicket ensuring that his front foot landed on a Kistler force platform during the delivery stride. A Photosonics camera, operating at $200 \mathrm{~Hz}$ filmed perpendicular to the plane of motion, while an overhead camera, at $100 \mathrm{~Hz}$, also filmed each trial. A large clock (to calculate film speed) and a reference marker of known length (to convert film measurements to real-life size) were included in the photographic field. Ample distance was available for the bowlers to use their normal run-up as the biomechanics laboratory, housing the force platform, opened onto an oval arena.

Force data were recorded from the Type 9281 Kistler force platform on a Schlumberger Instrumentation Recorder for later off-line analysis (bandwidth 0 $10 \mathrm{KHz}$ ). A shutter correlation pulse was encoded on both the film and the magnetic tape so that kinematic and temporal data from the film could be synchronized with the kinetic data from the force platform.

All bowlers kept a log book of their bowling statistics both during practice and in matches during the season. These statistics included the number of overs bowled, the length of bowling spells and match performances.

\section{Treatment and analysis of the data}

All cricket-related injuries during the season were assessed by a sports physician, who used computerized tomography to assist in the diagnosis of spinal injuries. At the completion of the season all players were grouped according to their injury status. Group 1 comprised those bowlers who had suffered a fracture to a vertebra(e), while Group 2 consisted of those bowlers who had experienced a soft tissue injury to the back that caused them to miss at least one match. Those players who remained injury free or who suffered only mild soft tissue injuries were categorized as Group 3.

After the film was processed the highest velocity trial, providing the front foot landed completely on the force platform during the delivery stride, was selected for analysis. The co-ordinates of selected features (the anatomical link system and ball) were recorded using a Numonics 224 digitizer and stored on disc in a DEC System 10 computer. An automatic smoothing procedure developed by Wood and Marshall ${ }^{12}$ and similar to the procedures reported by Wells and Winter ${ }^{13}$ was used so that the motion of different anatomical landmarks and body segments could be smoothed at varying frequencies. Time derivative information was determined using finite difference calculus.

The DEC System 10 computer was then used to convert the analogue force traces that had been recorded on magnetic tape into a digital time series. Values for each selected bowling trial (sampled at $500 \mathrm{~Hz}$ ) were then displayed so that scalar values could be determined at selected points in the bowling action.

A one-way analysis of variance was used to test for significant differences between the three groups $(P<0.05)$. Where a significant $\mathrm{F}$ ratio for main effects was recorded a Scheffe post hoc analysis was used to determine which groups were in fact different.

\section{Definition of terms}

Front-on bowling action A shoulder alignment of greater than $200^{\circ}$ at back or front foot impacts, where the right hand horizontal is a line drawn through the leading shoulder parallel with the pitch.

Side-on bowling action A shoulder alignment of less than $190^{\circ}$ at back foot or front foot impacts.

Delivery stride The bowling action between the back foot strike and ball release.

\section{Results and discussion}

\section{Incidence of injury}

The fact that 38 per cent of the fast bowlers sustained at least one disabling injury during the 1986-1987 season emphasizes the traumatic nature of fast bowling. Eleven per cent of the players sustained a stress fracture to the lumbar (one at L4 and seven at L5) and/or sacral vertebrae (one at an incompletely sacralized S1), while 27 per cent sustained a soft tissue injury to the back region that caused them to miss at least one match. Another player, although not included in the above statistics, sustained a stress fracture of the tibia.

Three of the nine bowlers diagnosed with a stress fracture to the lumbar vertebra were found to be genetically predisposed to pars defects, while six subjects developed pars fractures probably as a result of the repetitive stress of bowling (Figure 1). The incidence of stress fractures ( 11 per cent) in this study is less than the 50 per cent reported by Payne et al. for 12 A grade fast bowlers followed over a five year period ${ }^{2}$. Fractures occurred most frequently in the fifth lumbar vertebra (70 per cent) which supports the findings of Cyron and Hutton ${ }^{14}$ and Eisenstein ${ }^{15}$. They described the fifth lumbar vertebra as the most vulnerable to stress fracture due to the abrupt change in flexibility between the spinal column and the pelvis and, in the event of a weak multifidus muscle, the neural arch of this vertebra is subjected to greater bending forces.

The mean age for the bowlers in this study also increased their vulnerability to fracture as at this age, the human intervertebral disc is relatively elastic. This allows much of the intervertebral shear force to reach the facet joints, placing undue stress on the pars interarticularis. Furthermore, complete ossification of 


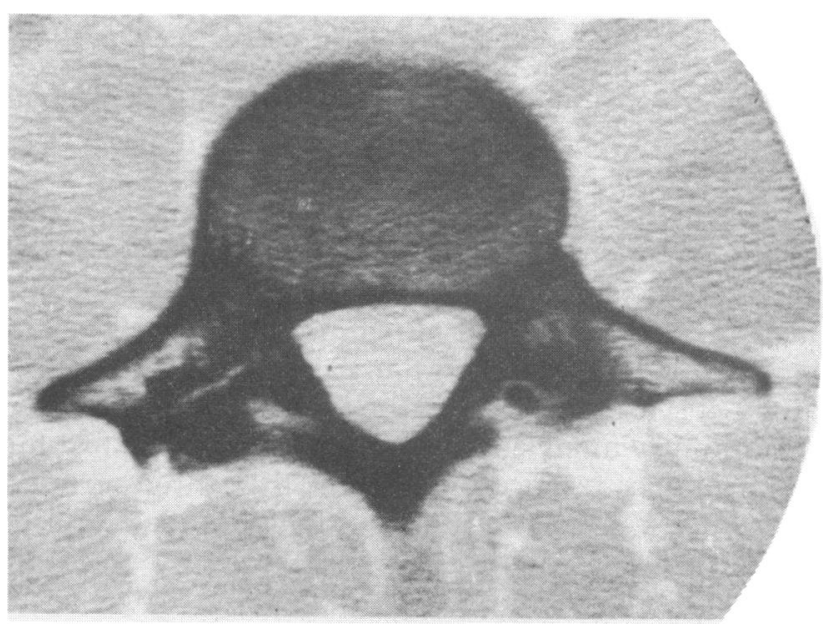

Figure 1. Stress fracture in the right pars interarticularis L5 shown on CT scan

\section{PHYSICAL CHARACTERISTICS TECHNIQUEFAULTS}

Posture

*Flat feet

\section{Strength}

*Reliance on upper body strength to generate ball velocity
BFI

*Excessive shoulder rotation to attain a side-on position between $\mathrm{BFI}$ and FFI

\section{FFI}

*Higher release position caused by larger knee and hip angles (less dissipation of force)

\section{OVERUSE \\ *Too many overs \\ *Too long a single spell \\ BACK INJURY}

Figure 5. Model of predisposing factors to back injury in fast bowling

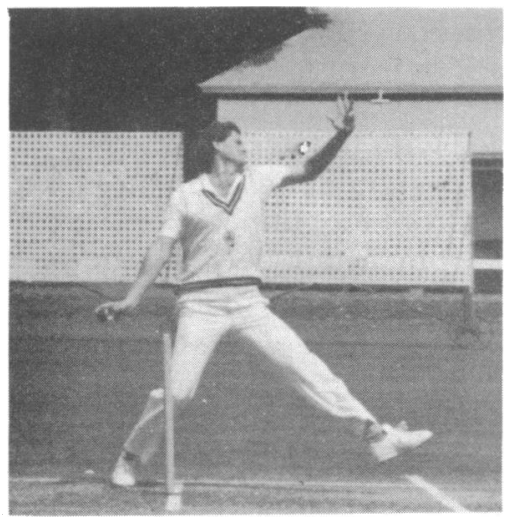

Figure 2A. Body position at BFI, sideon orientation

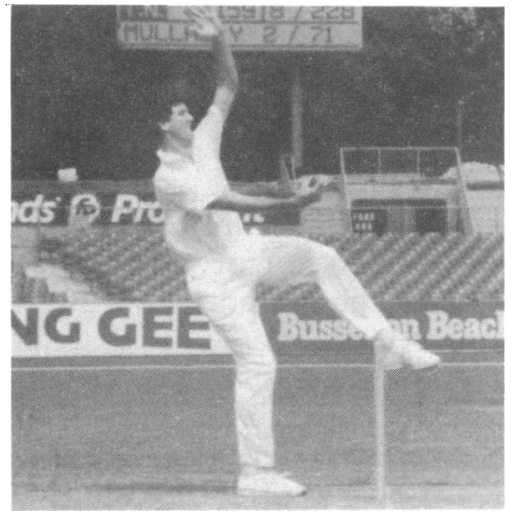

Figure 2B. BFI, front-on orientation



Figure 3A. FFI, side-on orientation

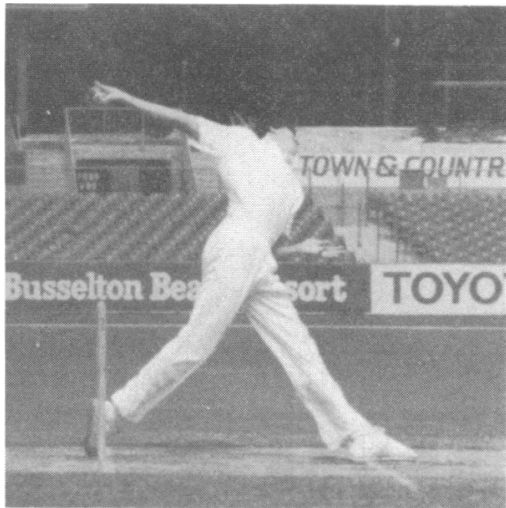

Figure 3B. FFI, front-on orientation

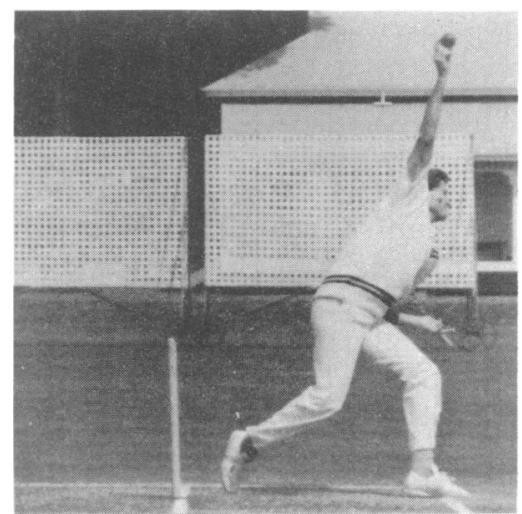

Figure 4A. Release, side-on orientation

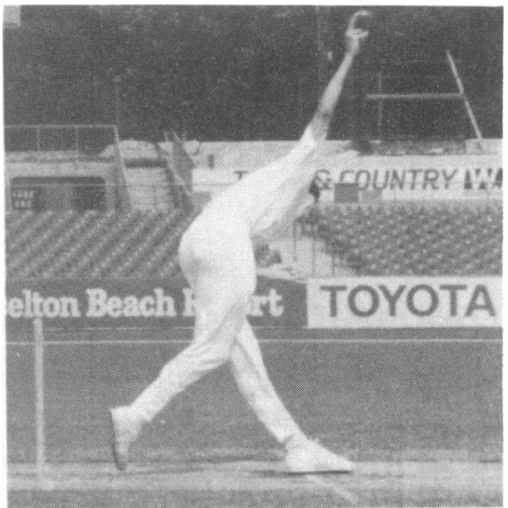

Figure 4B. Release, front-on orientation the neural arch has not occurred, making the pars interarticularis more vulnerable under repetitive stress. It has been shown that growth cartilage is less resistant to repetitive stresses than adult cartilage ${ }^{16}$.

The fact that fast bowling requires repetitive lateral flexion of the trunk, and that predominantly front-on bowlers adopt a hyperlordotic posture during the delivery action, further increases the possibility of developing a stress fracture in the lumbar region ${ }^{17,18}$. Rovere stated that few athletes with adequate abdominal and paraspinal muscle strength experienced back injuries as a result of poor posture but rather, the injuries tended to be related to movements within their sport particularly if a hyperlordotic posture was adopted in the activity ${ }^{19}$. The fast bowlers in this study generally adopted a hyper-extended spine as they prepared to deliver the ball. This may have been a contributing factor in the high incidence of back injuries diagnosed for the fast bowlers in this study.

\section{Kinanthropometric variables}

The only kinanthropometric variable that significantly differentiated the groups was the level of the longitudinal arch of the foot, where bowlers with a low arch were more likely to develop a stress fracture. The foot 
arches are designed to help absorb impact forces. Therefore, a foot with a low arch may not be as capable of absorbing these forces (mean vertical ground reaction force $(\mathrm{GRF})=5.43$ body weights $(\mathrm{BW})$ ) as effectively as a foot with a medium or high arch. The subject who developed a stress fracture in the tibia of the front bowling limb not only had a low foot arch, but also generated the highest maximum vertical GRF at front foot impact (FFI) of $8.96 \mathrm{BW}$.

\section{Physiological variables}

The ratio of quadriceps torque to hamstring torque $(1.77: 1)$ was similar to previously reported values ${ }^{20}$, while the torque differences for those muscle groups between preferred and non-preferred lower limbs were less than 10 per cent a level above which research has indicated may lead to injury ${ }^{21}$. The relationship between quadriceps torque in the non-preferred (front limb) and stress fractures of the lower back can not be explained.

Higher levels of shoulder depression strength (preferred limb) and shoulder horizontal flexion strength (preferred limb) were significantly related to back injury. Bowlers with greater upper body strength may have exerted higher twisting forces on the spine in their efforts to achieve optimal ball velocity. This may contribute to the development of back injuries.

Hoshina had previously reported a significant relationship between upper body strength and spondylolysis in male high school and university athletes ${ }^{22}$. The fact that the bowlers in this study used a front-on action may also mean that a reliance on excessive upper body strength rather than using the rotation of the shoulders as part of a gradual summation of velocity may have occurred with these bowlers. No other physiological variables were significantly related to the onset of back injuries.

\section{Biomechanical variables}

The delivery action of a fast bowler is a complex series of movements that can be separated into discrete phases. The discussion will be sub-divided into the run-up to back foot impact (BFI), the delivery stride, and release.

\section{Run-up to back foot impact}

The mean approach velocity of $4.95 \mathrm{~m} \mathrm{~s}^{-1}$, which was higher than the mean velocity for Australian national level bowlers who used a side-on technique ${ }^{23}$ suggested that these bowlers would have trouble adopting a side-on bowling action. No significant relationship was found between approach velocity, angle of the back foot, or alignment of the shoulders at BFI (Figures $2 A$ and $B$ ).

The mean shoulder alignment of $219^{\circ}$ shows that this sample of fast bowlers indeed had a front-on shoulder orientation (alignment of greater than $200^{\circ}$ ) for the delivery stride which Lillee ${ }^{24}$ and Elliott et al. ${ }^{25}$ both stated was a contributing factor to back injuries.

However, of the nine subjects who recorded a shoulder alignment of less than $190^{\circ}$ (side-on orientation), only one sustained a back injury. In contrast, of the 15 subjects who had an alignment of greater than $240^{\circ}$ (excessively front-on), eight sustained some form of back injury. It may then be implied that bowlers who recorded a shoulder alignment of greater than $240^{\circ}$ at BFI face a greater risk of back injury than do bowlers who align their shoulders more parallel with the wicket.

\section{The delivery stride}

At front foot impact (FFI) (Figures $3 A$ and $B$ ), the mean shoulder alignment of $202.7^{\circ}$ indicated that these bowlers rotated to decrease the alignment angle of the shoulders between BFI and FFI. A significant difference was recorded between Group 1 and Group 3 for shoulder rotation between BFI and the position of maximum rotation with Group 1 rotating more than Group 3. Of the bowlers who rotated more than $40^{\circ}$ from the shoulder alignment at BFI to a position closer to parallel to the wicket at FFI $(n=17$, Figures $2 B$ to $3 B)$, six sustained a stress fracture and seven sustained a soft tissue injury to the back.

The bowlers who were not injured during the season only rotated $16^{\circ}$ from BFI to the position of maximum rotation and $15.7^{\circ}$ from BFI to FFI. These results suggest that excessive rotation of the shoulders from the position at BFI to a more side-on position during the delivery stride has the potential to cause injury.

No significant relationship was determined between the peak vertical or horizontal ground reaction force (GRF) at FFI and back injuries. However, these values of $5.43 \mathrm{BW}$ (vertical) and $-2.45 \mathrm{BW}$ (horizontal) were higher than peak values of $4.7 \mathrm{BW}$ and $-1.7 \mathrm{BW}$ respectively reported for elite fast bowlers ${ }^{23}$.The GRFs generated at FFI are transmitted by the bones, tendons and muscle to the knee and hip joints and absorbed by the body ${ }^{26}$. Provided that the spine is erect, which is more the case in the side-on bowling position, the intervertebral compressive force developed through impact is more likely to be resisted by the bowler's intervertebral discs. However, in a lordotic posture or when the spine is hyper-extended, as with a front-on bowling technique, the facet joints may bear more of this compressive force. While higher forces at FFI were not significantly related to back injuries, the level of the GRFs may certainly predispose a bowler to back injuries if he is required to bowl for a long spell.

While the back injuries diagnosed in this study occurred at varying times throughout the season, it was evident that the amount of bowling was also related to injury. Of the 32 subjects who bowled in more than 17 matches, the mean number for the group, 19 sustained a stress fracture or back injury. Forty-one subjects reported bowling in excess of 10 overs in a spell during the season (mean for group $=8.5$ overs), and of this number, 27 reported abnormal lower back pain the following day. Micheli ${ }^{1,27}$, and Harvey $^{28}$ suggested that repetitive activity appeared to be a common factor in many over-use injuries. Bowling too many overs in any single spell and/or bowling too many spells may lead to back injuries to the bowler.

\section{Release}

The release height (Figures $4 A$ and $B$ ), whether expressed as an absolute value or as a percentage of standing height significantly differentiated the stress fracture group from the bowlers who were not injured. That is, the subjects who sustained stress fractures tended to 
deliver the ball from a greater height than the bowlers who were not injured during the season. Similarly, higher shoulder and hip angles were general characteristics of those bowlers who suffered a stress fracture during the season. The non-injured bowlers decreased their hip, shoulder and front knee angles at FFI possibly to dissipate GRFs, but in doing so, they reduced the release height of the ball.

In contrast, those bowlers who suffered stress fractures tended to keep their front knee joint extended and the trunk more upright (causing a larger shoulder angle) which may have made it more difficult to dissipate the large GRFs, than was the case for those bowlers who flexed the front lower limb.

\section{Conclusion}

The incidence of injury (38 per cent) and particularly back injuries (nine stress fractures of the lumbar or sacral vertebrae, 22 soft tissue back injuries) diagnosed for young bowlers highlights the frequency of fast bowling injuries. There was no single cause but rather a combination of factors which may have predisposed these bowlers to injury. One or more of inadequate physical and/or physiological attributes, postural defects, poor bowling technique or the high physical demands placed on young fast bowlers were predisposing factors to injury. (Figure 5).

Bowlers who rotated their shoulders more than $40^{\circ}$ from the shoulder alignment at BFI to a more side-on position at FFI were more likely to sustain back injuries. It is therefore important for bowlers to adopt a side-on shoulder orientation at BFI, rather than trying to alter alignment from a front-on position at BFI to a more side-on position at FFI.

Those bowlers who adopt a front-on position of the lower body at BFI must not therefore adopt a side-on upper body orientation, but must use a front-on upper body orientation.

Bowlers who delivered the ball from a high release height relative to their standing height seemed to less effectively dissipate GRFs at FFI and therefore had more of a chance of sustaining stress fractures of the lower back.

Too much reliance on upper body strength rather than good technique may also predispose a young bowler, who is attempting to bowl fast, to back injury. A sudden escalation in training frequency and/or duration or length of spell(s) during a match may also predispose the bowler to back injuries.

Further explanation for the high incidence of injuries to the young fast bowlers in this study was that the age of the bowlers (mean $=16.8$ years) may have predisposed them to back injuries as the neural arches of their lumbar vertebrae may not have completely ossified.

\section{Acknowledgements}

The authors thank the Australian Sports Commission for their support of this project.

\section{References}

1 Micheli, L.J. Overuse injuries in children's sports: The growth factor Orthop Clin North Am 1983, 14(2), 337-357

2 Payne, W.R., Hoy, G. and Carlson, J.S. What research tells the cricket coach Sports Coach 1987, 10(4), 17-22

3 Jackson, D.W., Wiltse, L.L. and Cirincione, R.J. Spondylolysis in the female gymnast Clin Orthop 1976, 117, 68-73
4 McBryde, A.M. Stress fractures in athletes I Sports Med 1975, 3, 212-217

5 Carter, J.E.L. and Heath, B.H. Somatotype methodology and kinesiological research Kinesiological Review 1971, 10-19

6 Behnke, A.R. and Wilmore, J.H. 'Evaluation and Regulation of Body Build and Composition' PrenticeHall Inc., 1974, Englewood Cliffs

7 Clarke, H.H. and Clarke, D.H. 'Developmental and Adapted Physical Education', Prentice-Hall Inc., 1963, Englewood Cliffs

8 Leighton, J.R. A simple objective and reliable measure of flexibility Research Quarterly 1942, 13, 205-216

9 AAHPER 'Health Related Physical Fitness Test Manual' AAHPER Publications 1976, Reston, Virginia

10 Johnson, P.B., Updyke, W.F., Stolberg, D.C. and Schaefer, M. 'Physical Education - A Problem-Solving Approach to Health and Fitness' Holt, Rinehart and Winston Inc., 1966, New York

11 Pyke, F. and Smith, R. 'Football - The Scientific Way,' University of Western Australia Press, 1975, Perth

12 Wood, G.A. and Marshall, R.N. The accuracy of DLT extrapolation in three dimensional motion analysis J Biomech 1986, 19(9), 781-785

13 Wells, R.P. and Winter, D.A. 'Assessment of Signal and Noise in the Kinematics of Normal, Pathological and Sporting Gaits' Proceedings of the Special Conference of the Canadian Society of Biomechanics, 92-93, 1980, London, Canada

14 Cyron, B.M. and Hutton, W.C. The fatigue strength of the lumbar neural arch in spondylolysis J Bone Joint Surg 1978, 60B(2), 234-238

15 Eisenstein, S. Spondylolysis J Bone Joint Surg 1978, 60B(4), 488-494

16 Cyron, B.M. 'Mechanical Factors in the Etiology of Spondylolysis' (PhD thesis, 1977) The Polytechnic of Central London, London, England

17 Krenz, M. and Troup, J.D.G. The structure of the pars interarticulares of the lower lumbar vertebrae and its relation to the etiology of spondylolysis J Bone Joint Surg 1973, 55-B(4), 735-741

18 Rossi, F. Spondylolysis, spondylolithesis and sports J Sports Med 1978, 18(4), 317-340

19 Rovere, G.D. (1987) Low back pain in athletes The Physician and Sportsmedicine 1987, 15(1), 105-117

20 Davies, G.J. 'Isokinetics in Clinical Usage' Second Edition S and S Publishers, 1985, La Crosse, Wisconsin

21 Gleim, G.W., Nicholas, J.A. and Webb, J.N. Isokinetic evaluation following leg injuries The Physician and Sportsmedicine 1978, 6(8), 74-82

22 Hoshina, H. Spondylolysis in athletes The Physician and Sportsmedicine 1980, 8(9), 75-81

23 Elliott, B.C. and Foster, D.H. A biomechanical analysis of the front-on and side-on bowling techniques Journal of Human Movement Studies 1984, 10, 83-94

24 Lillee, D.K. 'The Art of Fast Bowling' Collins Publishing Company, 1977, Sydney

25 Elliott, B.C., Foster, D.H. and Gray, S. (1986) Biomechanical and Physical Factors Influencing Fast Bowling Australian Journal of Science and Medicine in Sport 1986, 18(1), 16-21

26 Nigg, B.M. 'The Load on the Lower Extremities in Selected Sports Activities,' 'Collected Papers on Sports Biomechanics' (ed. G. Wood) Department of Human Movement and Recreation Studies Publications, 1983, 62-72

27 Micheli, L.J. 'Lower Extremity Injuries: Over-use Injuries in the Recreational Adult Athlete' In Cantu R.C. (ed) 'The Exercising Adult' Collamore Press, 1982, Lexington, Massachusetts

28 Harvey, J.S. Overuse syndromes in young athletes Clin Sports Med 1983, 2(3), 595-607

Editor's note Full statistical data is available, on request, from the authors. 